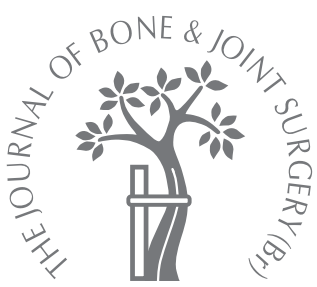

\title{
A novel method of accurately calculating the radiological magnification of the hip
}

\author{
R. J. King, \\ P. Makrides, \\ J. A. Gill, \\ S. Karthikeyan, \\ S. J. Krikler, \\ D. R. Griffin \\ From University \\ Hospitals Coventry \\ and Warwickshire \\ NHS Trust, Coventry, \\ England
}

\author{
R. J. King, FRCSEd(Trauma \& \\ Orth), Locum Consultant \\ Orthopaedic Surgeon \\ P. Makrides, MRCS, Specialty \\ Registrar \\ S. J. Krikler, BSc, PhD, \\ FRCS(Orth), Consultant \\ Orthopaedic Surgeon \\ University Hospitals Coventry \\ and Warwickshire NHS Trust, \\ Clifford Bridge Road, Coventry \\ CV2 2DX, UK.
}

J. A. Gill, BSC(Hons), MSc

Medical Student

- S. Karthikeyan, MBBS

MRCS, Clinical Research Fellow

D. R. Griffin, MA,

MPhil(Epid), FRCS(Tr \& Orth)

Professor

Clinical Sciences Research

nstitute

Warwick Medical School,

University Hospitals Coventry

and Warwickshire NHS Trust,

Clifford Bridge Road, Coventry

CV2 2DX, UK.

Correspondence should be sent

to Mr R. J. King; e-mail:

j.hall@warwick.ac.uk

(C)2009 British Editorial Society

of Bone and Joint Surgery

doi:10.1302/0301-620X.91B9.

$22615 \$ 2.00$

$J$ Bone Joint Surg $[\mathrm{Br}]$ 2009;91-B:1217-22.

Received 18 March 2009;

Accepted after revision $12 \mathrm{May}$

2009

\begin{abstract}
We have developed a novel method of calculating the radiological magnification of the hip using two separate radio-opaque markers. We recruited 74 patients undergoing radiological assessment following total hip replacement. Both the new double marker and a conventional single marker were used by the radiographer at the time of x-ray. The predicted magnification according to each marker was calculated, as was the true radiological magnification of the components. The correlation between true and predicted magnification was good using the double marker $(r=0.90, n=74, p<0.001)$, but only moderate for the single marker $(r=0.50$, $n=63, p<0.001$ ). The median error was significantly less for the double marker than for the single $(1.1 \%$ vs $4.8 \%, p<0.001)$. The double marker method demonstrated excellent validity (intraclass correlation coefficient $=0.89$ ), in contrast to the single marker $(0.32)$.

The double marker method appears to be superior to the single marker method when used in the clinical environment.
\end{abstract}

Accurate pre-operative planning for total hip replacement requires that the magnification of the radiograph is known. The recent widespread introduction of digital radiography throughout the United Kingdom has highlighted this need, not least because the image size varies according to the dimensions of the digital display. Templating software permits instant resizing of digital templates to match precisely the magnification of the radiograph. This magnification is generally determined using a single scale marker (such as a disc or sphere) of known dimensions, positioned in the same coronal plane as the hip joints. Although there are reports ${ }^{1-4}$ suggesting that such markers may permit highly accurate calculation of magnification, this has not been our experience. In our department it has been standard practice to include such a scale marker on the pre-operative radiograph of all patients presenting for a primary hip replacement. The marker consists of a $25.4 \mathrm{~mm}$ sphere mounted on a flexible arm, and is positioned by a radiographer trained in its use. We have often observed, however, that templating for hip replacement is often inaccurate when these 'scaled' radiographs are used. A departmental audit revealed that the marker was malpositioned in a quarter of cases. In view of these concerns, we have developed a new type of radiographic scale marker, one which we believe will be highly accurate in all patients, easy to apply correctly, and acceptable to patients. In this paper we first describe the theory and design of the new marker, followed by the results of a direct comparison of the new method with the standard single marker method.

\section{Materials and Methods}

Design of the new radiological scaling method.

Our primary objective was to develop a radiological marker that could be applied correctly without having to first identify the coronal plane of the hips. The new method actually uses two separate radiological markers: one behind the pelvis and the other in front, as the patient lies supine. These positions are unambiguous and can be reliably identified in all patients. Figure 1 illustrates the arrangement of these markers, and shows that they may be used to determine the magnification of the hip by means of a simple calculation. This calculation requires that the relationship of the coronal plane of the hips to the anteroposterior (AP) dimension of the supine patient at the level of the hips is known. We term this the 'coronal plane ratio' $\left(\mathrm{R}_{\mathrm{cp}}\right)$.

The first part of the study was to determine an approximate value for $R_{c p}$. In order to do this, we identified 50 patients who had recently undergone a pelvic CT scan. The digital images were retrieved from the hospital Patient Archiving Communication System (PACS), 

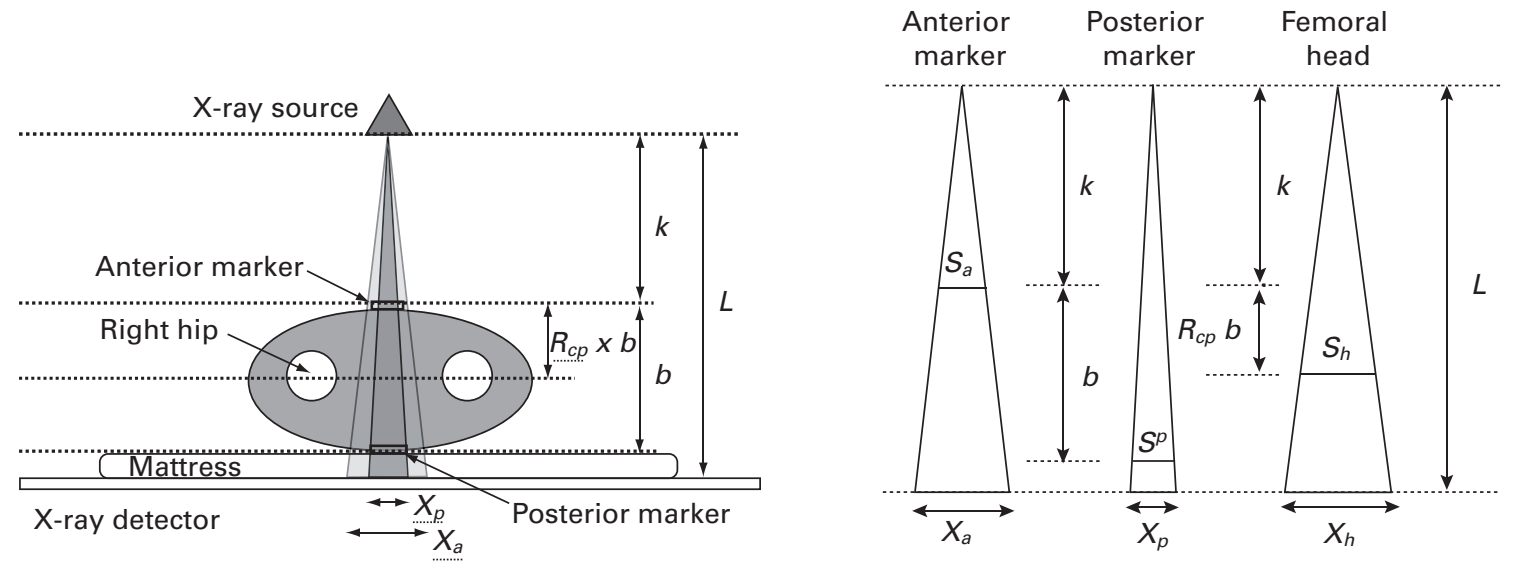

Fig. 1

Diagrams showing the theory of the double marker method.

and in each case the transverse slice that best displayed the centre of the femoral heads was identified. Using this software, a line was drawn connecting the centre of each femoral head to indicate the coronal plane of the hips (Fig. 2). Next, a line was marked perpendicular to this, passing through the midline of the patient from the anterior to posterior skin surfaces (Points A and E, respectively), indicating the mid-sagittal plane. The length of line $\mathrm{AC}$ was measured, where $\mathrm{C}$ was the point where the lines crossed. $R_{c p}$ was defined as the length $A C$ divided by length $A E$. The mean value for the sample was then calculated. In order to determine whether $R_{c p}$ was related to the patient's body habitus, the thickness of the anterior and posterior subcutaneous adipose layers was also measured (lengths $\mathrm{AB}$ and DE, respectively).

The design of the new double marker was as follows: the posterior marker was made of a $10 \mathrm{~mm}$ thick radiolucent foam mat incorporating 20 steel rods, each exactly $25.4 \mathrm{~mm}$ long. The rods were arranged in a vertical row down the centre of the mat, orientated transversely at $20 \mathrm{~mm}$ intervals. The anterior marker consisted of five $25.4 \mathrm{~mm}$ precision stainless steel ball bearings, secured to a strip of adhesive tape at approximately $50 \mathrm{~mm}$ intervals. The posterior marker mat was placed on the x-ray table, and the patient was asked to lie supine with their pelvis on the mat so that the marker rods would appear in the midline on the radiograph. The anterior marker strip was then placed longitudinally over the patient's suprapubic area, again approximately in the midline. Any bulky clothing that tended to lift the marker away from the patient's skin was removed. There were two reasons for using multiple anterior and posterior markers, first to ensure that at least one anterior ball and one posterior rod would be located at the level of the hips, and second to minimise the chances that the anterior and posterior markers would completely obscure each other on the radiograph. It should be pointed out that the posterior markers were affixed to the under-

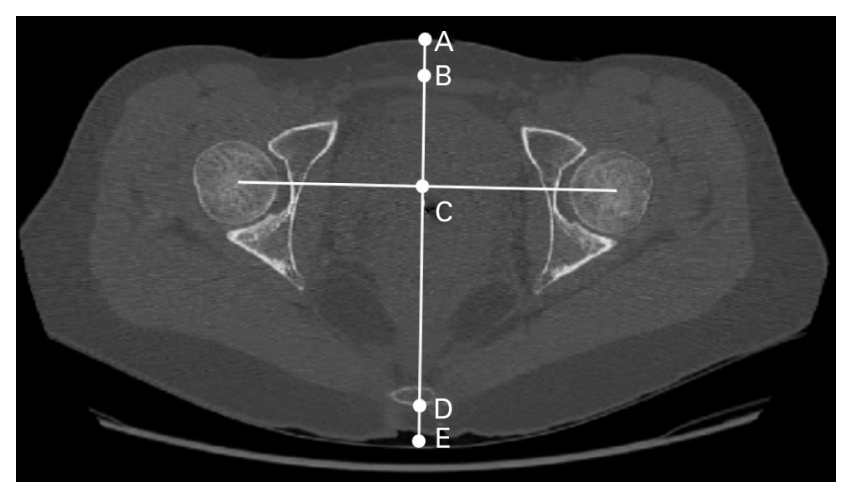

Fig. 2

CT scan of both hips, showing the measurements made to calculate the coronal plane ratio.

surface of the mat. This was to ensure that they were approximately $10 \mathrm{~mm}$ away from the patient's skin, to mirror the situation with the anterior markers; the projected circumference of the anterior marker was approximately half the diameter of the marker ball above the skin surface.

In order to calculate the radiological magnification of the hips, the anterior marker ball and the posterior marker rod closest to the hip joints are identified on the radiograph (Fig. 3). The diameter of the anterior marker $\left(\mathrm{X}_{\mathrm{a}}\right)$ and the length of the posterior marker $\left(\mathrm{X}_{\mathrm{p}}\right)$ are measured on the radiograph. The predicted magnification of the hips $\left(\mathrm{M}_{\mathrm{double}}\right)$ is then calculated as follows:

$$
\mathrm{M}_{\text {double }}=\frac{100 \%}{\left(\frac{S_{\mathrm{a}}}{\mathrm{X}_{\mathrm{a}}}\right)+\mathrm{R}_{\mathrm{cp}}\left(\frac{\mathrm{S}_{\mathrm{p}}}{\mathrm{X}_{\mathrm{p}}} \frac{S_{\mathrm{a}}}{\mathrm{X}_{\mathrm{a}}}\right)}
$$

where $s_{a}$ and $s_{p}$ are the true dimensions of the anterior and posterior markers, respectively (Fig. 1). 


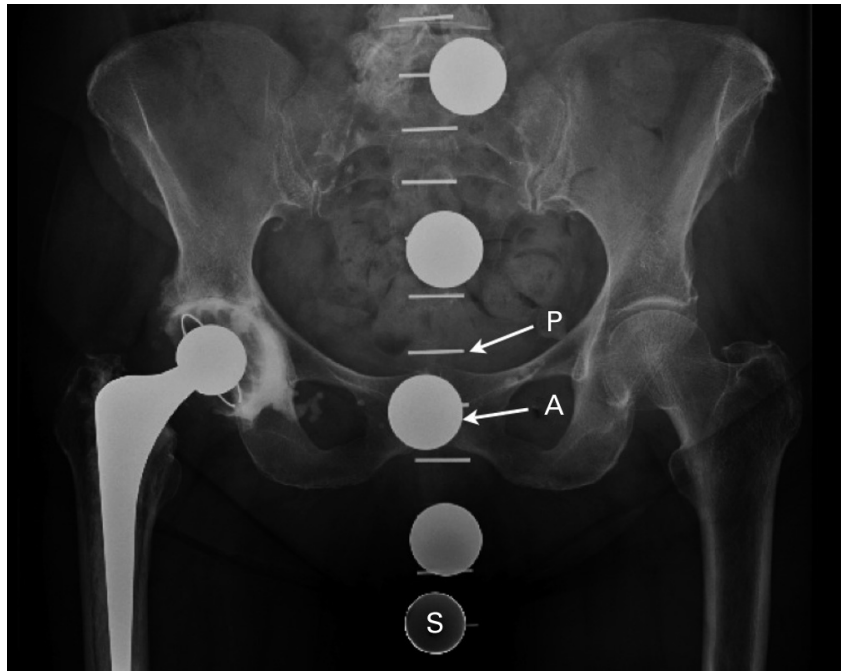

Fig. 3

Radiograph showing the double marker $(A=$ anterior marker ball closest to hips; $\mathrm{P}=$ posterior marker rod closest to hips). The single marker is also visible between the thighs (S).

Comparison of the double marker method with the single marker method. In the second part of the study, the new method was compared with the single marker previously used in our department. We recruited 74 patients undergoing routine radiological follow-up of a hip replacement. The operation notes were reviewed and the dimensions of the implanted components recorded. The patient was positioned supine, the markers for both methods were applied, and the radiograph taken. Of the 74 patients, 56 also had their height and weight measured and their body mass index (BMI) calculated.

As outlined above, the single marker consisted of a $25.4 \mathrm{~mm}$ precision stainless steel ball mounted on a flexible arm (OrthoMark, Rollesolutions Inc., Castro Valley, California). This ball was positioned in the coronal plane of the hips as determined by palpation of bony landmarks, either high up in the perineal area or at the level of the greater trochanter. All markers were applied by our departmental radiographers, and without any supervision by the authors.

The predicted magnification of the hips was determined for each method. The predicted hip magnification by the single marker method $\left(\mathrm{M}_{\text {single }}\right)$ was calculated by the following equation:

$$
M_{\text {single }}=\left(\frac{b_{\text {meas }}}{b_{\text {true }}}\right) \times 100 \%
$$

where $b_{\text {meas }}$ and $b_{\text {true }}$ are the measured true dimensions of the marker ball.

The actual (true) magnification of the hip $\left(\mathrm{M}_{\text {actual }}\right)$ was determined as follows:

$$
\mathrm{M}_{\text {actual }}=\left(\frac{\mathrm{P}_{\text {meas }}}{\mathrm{P}_{\text {true }}}\right) \times 100 \%
$$

where $\mathrm{p}_{\text {meas }}$ and $\mathrm{p}_{\text {true }}$ are the measured and true dimensions of the prosthesis.

Calculation of the true magnification was based on the femoral head size and corresponding radiological measurement, providing the maximal diameter of the head could be clearly identified and measured. In patients where the femoral head was obscured by an uncemented acetabular component, the diameter of the acetabular component was measured instead, providing it was known to be fully hemispherical without peripheral expansion. Patients with high hip centres were also excluded. In patients with bilateral hip replacements, the hip in the most anatomical position was chosen. Where both the replacements were suitable, the right hip was always used. We attempted to avoid any bias in the measurements by calculating the magnification for each marker method before calculating the true magnification.

In order to measure the inter-observer variability, an additional eight independent observers measured the radiological magnification of ten patients with an uncemented hemispherical acetabular component. The observers consisted of one orthopaedic consultant, six orthopaedic research fellows, and one foundation-year doctor. Radiographs of patients with uncemented acetabular components were used to ensure that the observers were truly blind to the actual dimensions of the components; although the size of the prosthetic femoral head may sometimes be determined simply from a familiarity with the implant, the diameter of the acetabular component is very variable, and cannot be guessed. Each observer measured the true magnification and the magnification as determined by the single and double markers. In order to determine the intraobserver error, all eight observers repeated the measurements after a minimum of seven days.

Statistical analysis. The Shapiro-Wilk test was used to determine whether the data was normally distributed. Pearson's product moment correlation coefficient was calculated to determine the relationship between the true and predicted magnification for each method. The intra-class correlation coefficient was calculated to describe the validity of each method as a predictor of magnification (model 2,1 as described by Shrout and Fleiss ${ }^{5}$ ). The errors for each marker were compared using the paired $t$-test. Inter- and intra-observer variability were also measured using the intra-class correlation coefficient. Statistical analysis was performed using SPSS (SPSS Inc., Chicago, Illinois), and significance was set at the $5 \%$ level. The statistical methodology was reviewed and approved by an independent statistician. 


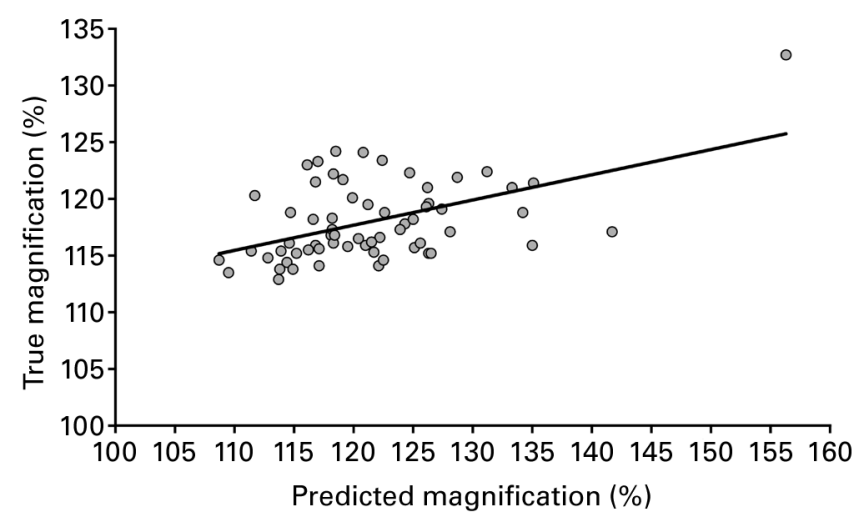

Fig. $4 a$

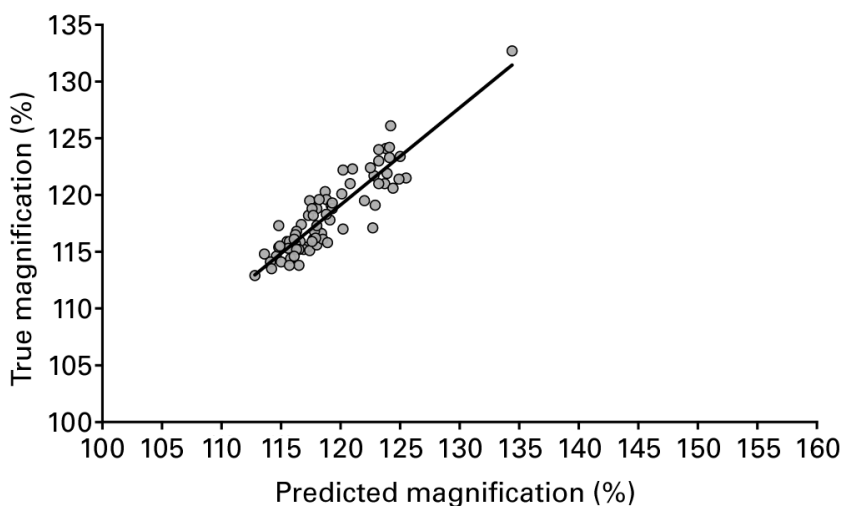

Fig. 4b

Graph showing the relationship between true magnification and the estimated magnification according to a) the single marker and b) the double marker method. Line of best fit shown.

\section{Results}

From the CT measurements in the first part of our study, the mean value for $\mathrm{R}_{\mathrm{cp}}$ was 0.45 (0.39 to 0.54 ) and was normally distributed $(\mathrm{p}=0.543)$. This mean value for $\mathrm{R}_{\mathrm{cp}}$ was subsequently used for the calculation of magnification using the double marker method. The correlations between $R_{c p}$, the thickness of the adipose layers and the anteroposterior distribution of adipose tissue were all poor $(\mathrm{r}<0.4)$.

The mean true magnification of the hip (as calculated from the known dimensions of the components) was $118.3 \%$ ( $112.9 \%$ to $132.7 \%)$.

The relationship between the true and predicted magnification using both marker methods is shown in Figure 4. There was good correlation using the double marker $(\mathrm{r}=0.90, \mathrm{n}=74, \mathrm{p}<0.001)$, but only moderate correlation for the single marker $(\mathrm{r}=0.50, \mathrm{n}=63, \mathrm{p}<0.001)$. The errors of each method are shown in Figure 5. The median error of the single marker was $4.8 \%(0.1 \%$ to $24.7 \%)$, but only $1.1 \%$ for the double marker $(0.5 \%$ to $5.6 \%)$; this difference was highly significant $(\mathrm{p}<0.001)$. If the magnification was assumed to be $118 \%$ in every case (the mean

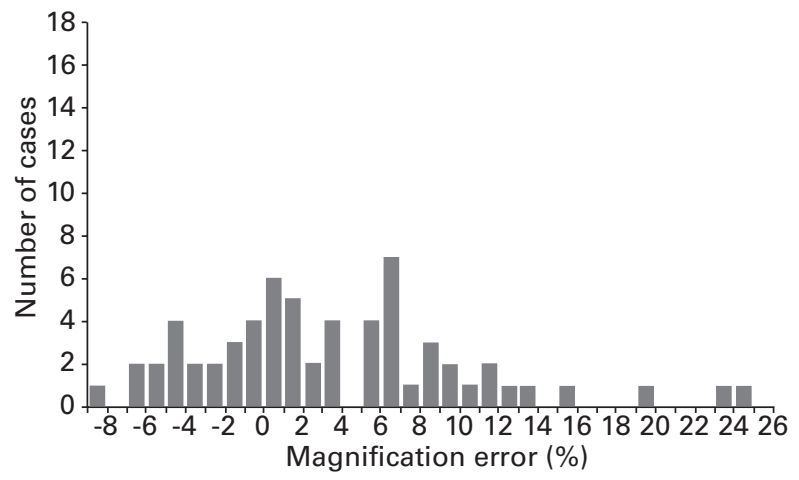

Fig. $5 a$

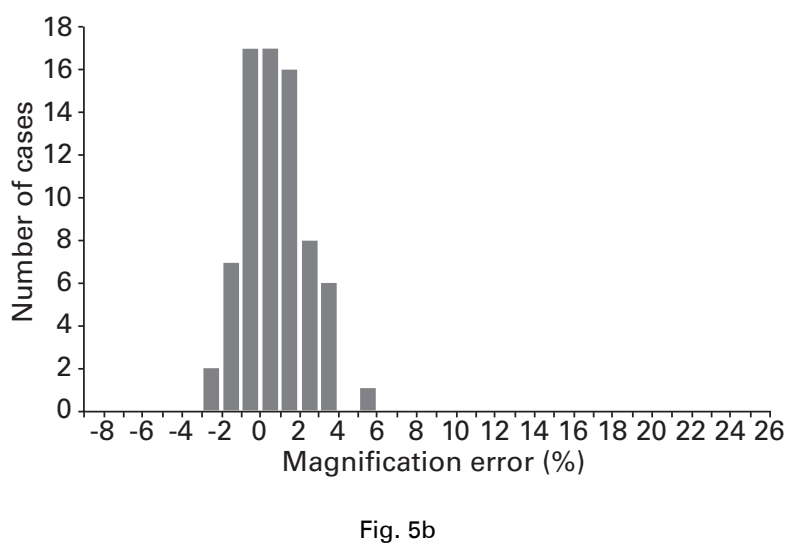

Graph showing the errors of predicted magnification according a) the single marker and b) the double marker method.

magnification in this series), the median error would be $2.4 \%(0.2 \%$ to $14.7 \%)$. Median values were calculated to minimise the effect of any outliers. The validity of the double marker method as a measure of true magnification was very good, with an intra-class correlation coefficient of 0.89 (95\% confidence interval (CI) 0.79 to 0.93 ). The validity of the single marker method was poor, with an intraclass correlation coefficient of only 0.32 (95\% CI 0.08 to 0.53 ). There was a slight trend for patients with a high $\mathrm{BMI}$ to have greater magnification, but correlation was not significant $(\mathrm{r}=0.19, \mathrm{n}=56, \mathrm{p}=0.16)$. The BMI was weakly correlated with the error of the double marker $(\mathrm{r}=0.38, \mathrm{n}=56, \mathrm{p}<0.01)$, but not the single marker $(r=0.22, n=46, p=0.18)$.

There was excellent observer reliability of all magnification measurements, as shown in Table I. The measurements of the eight independent observers were comparable to those of the main group of patients as assessed by just the one observer (RJK). The median error of the single marker method was $5.4 \%$ ( $0 \%$ to $18.1 \%$ ), but was only $1.3 \%$ for the double marker method $(0 \%$ to $7.9 \%)$. 
Table I. Intra-class correlation coefficients as a measure of observer variability (with $95 \%$ confidence intervals)

\begin{tabular}{llll}
\hline & $\begin{array}{l}\text { Measurement of true } \\
\text { magnification }\end{array}$ & $\begin{array}{l}\text { Magnification by single marker } \\
\text { method }\end{array}$ & $\begin{array}{l}\text { Magnification by double } \\
\text { marker method }\end{array}$ \\
\hline Inter-observer & $0.94(0.87$ to 0.98$)$ & $0.99(0.98$ to 1.00$)$ & $0.96(0.92$ to 0.99$)$ \\
Intra-observer & $0.90(0.85$ to 0.93$)$ & $0.96(0.94$ to 0.98$)$ & $0.94(0.91$ to 0.96$)$ \\
\hline
\end{tabular}

\section{Discussion}

It is important to be able to template the hip joint prior to performing a hip replacement. It is known that inaccurate sizing of components increases the risk of failure, ${ }^{6}$ and that careful templating can reduce the duration of the procedure and the risk of complications. ${ }^{7,8}$ Accurate templating is only possible when the radiological magnification of the hip is known.

Prior to the introduction of digital radiographs, templating of hip replacement was performed using transparent templates of fixed magnification, typically $115 \%$ to $120 \%$. All acute NHS hospitals in the United Kingdom now use digital radiographs, however, in line with current government policy. ${ }^{9}$ Physical templates are not suitable for digital images, as the size of the on-screen image varies with the dimensions of the monitor used, and can be enlarged or reduced on screen. For this reason, templating should now be performed using digital templates, as these can be easily resized to reflect the actual magnification of the radiograph.

As already mentioned, the most widely used method of calculating radiological magnification is to place a marker of known dimensions next to the patient at the time the radiograph is taken, and then measure this on the resulting image. The first description of such a device actually pre-dates the digital age. ${ }^{10}$ For this method to be accurate for the hip, the marker must be located as close to the hip joint as possible, and in the same coronal plane. This typically involves positioning a metal sphere or disc adjacent to the patient, either next to the greater trochanter or high up in the perineal area.

A number of papers have demonstrated that such single marker methods can be extremely accurate. Conn et al ${ }^{1}$ showed that by using a coin placed over the greater trochanter as a marker, the accuracy of femoral component sizing could be significantly improved. Wimsey et $\mathrm{al}^{2}$ described the use of a device incorporating coin markers positioned high up between the patient's thighs, with a mean error of magnification as low as $1.1 \%$. Bayne et $\mathrm{al}^{3}$ compared the OrthoMark device with an adhesive marker placed adjacent to the greater trochanter, and reported that the mean error of magnification was $<3 \%$. The et $\mathrm{al}^{4}$ used a marker ball placed in the coronal plane of the hips, with an error of magnification of only $1.1 \%$.

All of these single marker methods rely upon the marker being accurately located in the coronal plane of the hips. In order to locate this plane a number of techniques can be used, including palpation of the greater trochanter with internal rotation of the hips, and measurement of abdominal girth. ${ }^{4}$ There is no question that these single markers are accurate if placed correctly. It is crucial to recognise, however, that the good results reported in the literature are only obtained where the marker was positioned very carefully by a limited number of trained individuals making every effort to be as accurate as possible. We suspect that in the typical busy radiography department these markers will be positioned by a large number of people, and not with equal precision. In addition, although these single markers may appear to be perfectly located on the radiograph, they may actually be anterior or posterior to the correct coronal plane. The surgeon cannot identify the fact that the markers may be malpositioned and their use may therefore be misleading. A single marker may also be difficult to use in very large individuals, where the patient's pelvis is wider than the $\mathrm{x}$-ray detector, and so a marker placed adjacent to the greater trochanter is not imaged. Positioning of a marker high up between the patient's thighs may be the only option in this situation, but many patients and staff find this to be socially unacceptable.

In an attempt to address these problems, the use of a single marker placed on top of the pubic symphysis has been described. ${ }^{11}$ Because of the relatively constant relationship between the pubic symphysis and the coronal plane of the hips, the magnification of a marker over the symphysis can be used to predict the hip magnification. Unfortunately, palpation of the pubic symphysis may not be easy in the obese patient, and to locate it may require significant invasion of the patient's privacy. Also, the source-detector distance used for the radiograph must be kept constant, otherwise the relationship between the marker magnification and the hip magnification will change. Kulkarni et $\mathrm{al}^{12}$ have recently taken a similar approach, describing the use of an oversized calibration disc placed directly on the $\mathrm{x}$-ray cassette, thereby avoiding the need to identify bony landmarks. They also used CT scans to measure the distance of the coronal plane of the hips above the back of the patient. Their method, however, also requires that the radiographer sets the film-focus distance at a pre-determined level. In addition, they recommend that larger or smaller calibration discs should be used in larger or smaller patients. If the radiographer has not done both of these things correctly, the calibration disc will be misleading, but the clinician will be oblivious to this.

The double marker technique that we have developed offers a number of advantages. The markers are extremely simple to position. The radiographer has to ensure three things only: first, that the patient is lying centrally on the 
mat; second, that the anterior marker strip is placed in the midline over the suprapubic area; and third, that any bulky clothing has been removed. It is readily apparent from the radiograph when the markers have not been applied correctly, and the radiograph can be repeated. This minimises the chances of the surgeon being misled by a poorly positioned marker. In our study, only one radiograph needed to be repeated as the radiographer forgot to apply the anterior marker. The double marker is extremely accurate, and our results are comparable with the best reported elsewhere in the literature. ${ }^{2,4,13}$ The median error was only $1.1 \%$. In a patient with an acetabular diameter of $50 \mathrm{~mm}$, the median measurement error when templating for an uncemented acetabular component would be $0.55 \mathrm{~mm}$. In our series, the correct size of an uncemented acetabular would have been predicted in $77 \%$ of cases (error $<1 \mathrm{~mm}$ ), and no more than one size too large or small in the remaining $23 \%$ (error $1 \mathrm{~mm}$ to $3 \mathrm{~mm}$ ). If the single marker method had been used in the same population, the acetabular component would have been correctly predicted in $29 \%$ (error $<1 \mathrm{~mm}$ ), one size too large or small in $32 \%$ (error $1 \mathrm{~mm}$ to $3 \mathrm{~mm}$ ), and anywhere from two to six sizes too large or small in the remaining $39 \%$ (error $3 \mathrm{~mm}$ to $13 \mathrm{~mm}$ ). The precision of the double marker method does not depend on careful positioning of the marker by a limited number of individuals, and therefore remains extremely accurate even when applied by a diverse group of busy radiographers. The technique also demonstrates excellent observer reliability, and remains accurate when used by a group composed largely of junior surgeons. It is not influenced by the focal length or subject-detector distance: it is apparent from Figure 1 that these distances do not appear in the final magnification equation. This is because two markers are used. The accuracy of the double marker method does not seem to diminish significantly in obese patients, and the same markers are always used in exactly the same way in all patients, thus avoiding confusion. Finally, the double marker is not invasive of the patient's privacy, and is therefore more acceptable than a marker positioned high up between the thighs.

It is interesting that the estimation of magnification using the single marker method was even less accurate than simply assuming the magnification to be $118 \%$ (median error $4.8 \%$ and $2.4 \%$, respectively). It could be argued that more extensive training of the radiographers in the positioning of the single marker might have improved accuracy. Our study clearly suggests, however, that in everyday use, the precision of the single marker is poorer than the results reported in the literature. It appears to be very operator dependent, and our results demonstrate how inaccurate it may be in the 'real world' of clinical practice, with staff turnover and in a large, busy x-ray department. In contrast, the double marker method remains remarkably accurate.

The value of $R_{c p}$ (the ratio relating the coronal plane of the hips to the front and back of the pelvis) that we have used was determined from unselected patients undergoing CT scans of the pelvis. Clearly $\mathrm{R}_{\mathrm{cp}}$ may be different in patients with osteoarthritis of the hip. It may also be that $\mathrm{R}_{\mathrm{cp}}$ is not the same when the patient lies on a flat surface for radiography, as opposed to the dished surface of our CT scanner. We fully recognise that our CT-derived value of $\mathrm{R}_{\mathrm{cp}}$ is only an estimate of the ratio in the typical patient with osteoarthritis of the hip. The double marker method is, however, still extremely accurate, even when this approximate value of $R_{c p}$ is used. Further study is planned to determine a more accurate value of $R_{c p}$ and to investigate whether the ratio varies with BMI, age, race and gender. Potentially this will even further improve the precision of the double marker method. At the moment, however, we have no reason to doubt that our results can be applied to the general population.

In conclusion, we have developed a novel method of scaling a digital radiograph that permits accurate digital templating. The median error is $1.1 \%$, and the maximum error is $5.6 \%$. This double marker method is significantly more accurate than a conventional single marker when used in a clinical context. We believe that this technique could become the routine method of calculating radiological hip magnification in clinical practice, and recommend that it be used in preference to other previously described methods, particularly if templating software can be designed to reference from these markers automatically.

\section{Supplementary material}

ё Further information on the additional materials is available with the electronic version of this article on our website at www.jbjs.org.uk

No benefits in any form have been received from a commercial party related directly or indirectly to the subject of this article.

\section{References}

1. Conn KS, Clarke MT, Hallett JP. A simple guide to determine the magnification of radiographs and to improve the accuracy of preoperative templating. J Bone Joint Surg [Br] 2002;84-B:269-72.

2. Wimsey S, Pickard R, Shaw G. Accurate scaling of digital radiographs of the pelvis: a prospective trial of two methods. J Bone Joint Surg [Br] 2006;88-B:1508-12.

3. Bayne CO, Krosin M, Barber TC. Evaluation of the accuracy and use of x-ray markers in digital templating for total hip arthroplasty. J Arthroplasty 2009;24:407-13.

4. The B, Kootstra JW, Hosman AH, et al. Comparison of techniques for correction of magnification of pelvic X-rays for hip surgery planning. J Digit Imaging 2007;20:329-35.

5. Shrout PE, Fleiss JL. Intraclass correlations: uses in assessing rater reliability. Psychol Bull 1979;86:420-8.

6. Carter LW, Stovall DO, Young TR. Determination of accuracy of preoperative templating of noncemented femoral prostheses. J Arthroplasty 1995;10:507-13.

7. Eggli S, Pisan M, Muller ME. The value of preoperative planning for total hip arthroplasty. J Bone Joint Surg [Br] 1998;80-B:382-90.

8. The B, Djercks RL, van Ooijen PM, van Horn JR. Comparison of analog and digital preoperative planning in total hip and knee arthroplasties: a prospective study of 173 hips and 65 total knees. Acta Orthop 2005;76:78-84.

9. No authors listed. Delivering 21st century IT support for the NHS. National strategic programme. London: Department of Health, 2002.

10. Gorski JM, Schwartz L. A device to measure x-ray magnification in preoperative planning for cementless arthroplasty. Clin Orthop 1986;202:302-6.

11. Crooijmans HJ, Laumen AM, van Pul C, van Mourik JB. A new digital planning method for total hip arthroplasties. Clin Orthop 2009;467:909-16.

12. Kulkarni A, Partington P, Kelly D, Muller S. Disc calibration for digital templating in hip replacement. J Bone Joint Surg [Br] 2008;90-B:1623-6.

13. The B, Verdonschot N, van Horn JR, van Ooijen PM, Diercks RL. Digital versus analogue preoperative planning of total hip arthroplasties: a randomized clinical trial of 210 total hip arthroplasties. J Arthroplasty 2007;22:866-70. 